\title{
Performance Analysis of WiMAX for Mobile Applications
}

\author{
Margot Deruyck, Emmeric Tanghe, Wout Joseph, member IEEE, Daan Pareit, \\ Ingrid Moerman, member IEEE, and Luc Martens, member IEEE \\ Ghent University / IBBT, Dept. of Information Technology \\ Gaston Crommenlaan 8 box 201, B-9050 Ghent, Belgium \\ E-mail: emmeric.tanghe@intec.UGent.be
}

\begin{abstract}
The goal of this paper is to investigate the performance of a mobile WiMAX system for various settings of its physical-layer parameters and for realistic propagation channels. For this, a physical layer model of IEEE 802.16e is developed in software. Different propagation channels are implemented, such as the Rayleigh channel and the SUI channel models. Moreover, a tapped delay line channel model is developed for a specific area in Ghent (Belgium) using raytracing software. For this area, the maximum achievable range of a realistic mobile WiMAX system is found to be $2.7 \mathrm{~km}$. Additionally, the performance gain of MIMO systems with diversity is investigated. The diversity gain of a $2 \times 2$ MIMO Alamouti diversity scheme is found to vary between 3 and $5.5 \mathrm{~dB}$.
\end{abstract}

\section{INTRODUCTION}

The enormous growth of the internet causes a rising demand for high-speed internet access. WiMAX (worldwide interoperability for microwave access), a wireless technology for broadband communication based on the IEEE 802.16 standard [1], promises high datarates and gains popularity over wired technologies such as DSL (digital subscriber line) and cable. The main advantages of WiMAX over wired systems are its lower cost, deployment flexibility, and lower maintenance.

In [2], a performance analysis of WiMAX for fixed applications is presented. Herein, the simulated WiMAX system attains a maximum range of $3.4 \mathrm{~km}$ and a maximum bitrate of $13.1 \mathrm{Mbps}$. It is shown that, for a BER (bit error rate) of $10^{-6}$, the minimum required SNR (signal-to-noise ratio) increases with $6 \mathrm{~dB}$ when increasing the constellation (e.g., from QPSK to 16-QAM) and with $4 \mathrm{~dB}$ when the coding rate is raised. Furthermore, for the same BER, $2 \times 1$ MISO (multiple input single output) and 2x2 MIMO (multiple input multiple output) diversity schemes lower the required SNR with $4 \mathrm{~dB}$ and $7 \mathrm{~dB}$, respectively. In [3], a first estimation of fixed WiMAX performance is given: a maximum range of $3.2 \mathrm{~km}$ and a maximum bitrate of $12.7 \mathrm{Mbps}$ are found.

In this paper, we will analyse the IEEE 802.16e interface, operating in the $2-6 \mathrm{GHz}$ band. This interface is developed for mobile wireless applications and lets people communicate while they are moving. The goal of this paper is to investigate the performance of a mobile WiMAX system for various settings of its physical-layer parameters and for realistic propagation channels. For this, a model for the physical layer of IEEE 802.16e is developed in Simulink ${ }^{\circledR}$.
Different propagation channels are implemented, such as the Rayleigh channel and the SUI (Stanford university interim) channel models in [4]. Moreover, a realistic tapped delay line channel model is developed for a specific area in Ghent using the Wireless Insite ${ }^{\circledR}$ raytracing software. Achievable physical datarates are calculated from [5], and corresponding ranges are estimated from link budget calculations with the ErcegB path loss model [6]. Additionally, the performance gain of MIMO systems with diversity is investigated. Throughout the paper, comparison with the IEEE 802.16 standard for fixed applications is made using the model implemented in [2].

In Section II, the configuration of a mobile WiMAX transmitter and receiver is described. Section III presents the results of the performance simulations. In Section IV, mobile WiMAX performance is evaluated for a specific environment located in Ghent, Belgium. The propagation characteristics of this environment are simulated in the raytracing software. Finally, conclusions are drawn in Section V.

\section{Configuration}

For the performance analysis of mobile WiMAX, a model for the physical layer is built in Simulink ${ }^{\circledR}$. Fig. 1 shows the main blocks of the model. An input bitstream is generated from a stochastic Bernouilli process with probability 0.5 for the occurence of both zeros and ones. In a first step, this input data is randomized in order to avoid long runs of zeros or ones. The FEC (forward error correction) adds redundant bits to correct bit errors introduced by the channel and consists of a convolutional encoder and subsequent puncturing. The convolutional encoder has a constraint length of 7 and its native coding rate is $1 / 2$. Important to note, is that the realworld performance of mobile WiMAX will be better than the performance shown in this paper because of the use of a convolutional turbo encoder instead of the convolutional encoder we use in our investigation. The puncturing removes some of the parity bits of the convolutional encoder's output to produce higher coding rates. The interleaving block is implemented to reduce the occurence of error bursts. The repetition block repeats the bits two, four, or six times only when QPSK is used for the constellation. Next, the bits are mapped to the symbols of a constellation: IEEE 802.16e allows QPSK, 16-QAM, and 64-QAM as possible choices [5]. Mobile 
WiMAX uses the novel SOFDMA (scalable orthogonal frequency division multiple access) technique for the allocation of symbols in the time and frequency domain. SOFDMA supports a wide range of operating bandwidths to flexibly address the need for various spectrum allocation and application requirements. When the operating bandwidth increases, the FFT (fast Fourier transform) size (i.e., the number of subcarriers) is also increased to maintain a fixed subcarrier frequency spacing of $10.94 \mathrm{kHz}$. A number of subcarriers are used as pilots for channel estimation at the receiver's side and a cyclic prefix is added to the SOFDMA symbols to avoid ISI (inter symbol interference). How many and where the pilot subcarriers are inserted depends on the FFT size and the subcarrier permutation. For the subcarrier permutation, our model supports PUSC (partially used subcarrier) and FUSC (fully used subcarrier).

At the receiver's side, the channel estimation and compensation blocks estimate and compensate for the propagation channel's fading. The other receiver blocks perform the inverse operation of their corresponding transmitter blocks. The model is used for SISO (single input single output) as well as for MIMO simulations. For MIMO, 2x1 and 2x2 Alamouti spacetime block code diversity schemes are implemented [7].

For fixed WiMAX, a similar model was built in Simulink ${ }^{\circledR}$. The biggest difference between mobile and fixed WiMAX is the fact that fixed WiMAX uses OFDM (open frequency division multiplexing) instead of SOFDMA as mobile WiMAX. An OFDM symbol contains 256 subcarriers from which 8 subcarriers are reserved as pilot subcarriers. OFDM doesn't support scalability which makes the subcarrier frequency spacing variable. Furthermore, the repetition is also not supported in fixed WiMAX. However, we used the same convolutional encoder for fixed WiMAX as for mobile WiMAX, i.e. its length is 7 and its coding rate is $1 / 2$.

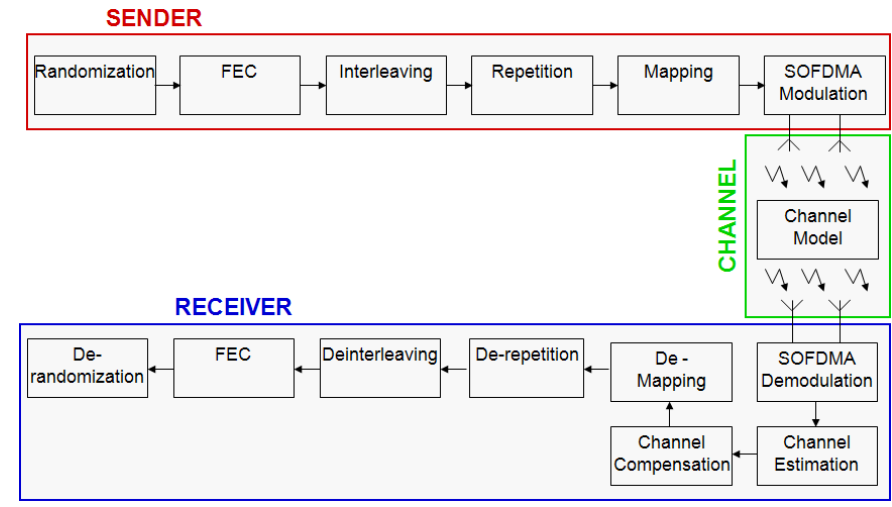

Fig. 1. Mobile WiMAX model

\section{Performance Results}

\section{A. Comparison of fixed and mobile WiMAX}

In this section, we investigate the difference in performance between fixed and mobile WiMAX. The results are presented as BER versus SNR graphs. Simulations are done for a range of increasing SNR values from $0 \mathrm{~dB}$ onwards until a BER of $10^{-6}$ is attained, which is assumed to stand for reliable communication. In this section, the following physical-layer parameters are chosen for the fixed and mobile WiMAX systems: QPSK for the constellation, a FEC coding rate of $3 / 4$, and a CP (cyclic prefix) length of $1 / 32$. Figs. 2 and 3 show BER versus SNR graphs for an AWGN (additive white Gaussian noise) channel and a Rayleigh channel, respectively. The simple AWGN channel consists of one propagation path to which normally distributed noise is added. Essentially, this channel constitutes a predominantly non-fading situation where most of the noise is created within the receiver itself. The Rayleigh channel creates multipath fading with three propagation paths with Rayleigh distributed path gains. The propagation paths have average path gains equal to $0,-10$, and $-15 \mathrm{~dB}$ with corresponding delays $0,0.25$ and $0.5 \mathrm{~s}$ [2]. Normally distributed noise is added to the combined signal from adding these three propagation paths in order to account for the receiver noise.

Figs. 2 and 3 show comparison results for both the PUSC and FUSC mode of mobile WiMAX, and for different FFT sizes. Fixed WiMAX is also shown for which the only supported FFT size is 256. For both the AWGN and Rayleigh channels, the BER versus SNR curve for fixed WiMAX is situated between the different curves for mobile WiMAX. For a FFT size of 128, mobile WiMAX outperforms fixed WiMAX because of the intercarrier interference. The higher the number of used subcarriers, the higher the intercarrier interference which results in a higher BER for the same SNR. Also, from Fig. 2, the BER versus SNR performance of the mobile PUSC and FUSC modes is almost identical in an AWGN channel. For the Rayleigh fading case in Fig. 3, certain mobile WiMAX modes show lower SNR requirements for a constant BER when compared to fixed WiMAX in the interesting area on the right where the BER is low. The better performing mobile modes are PUSC with FFT sizes of 128 and 512, and FUSC with an FFT size of 512.

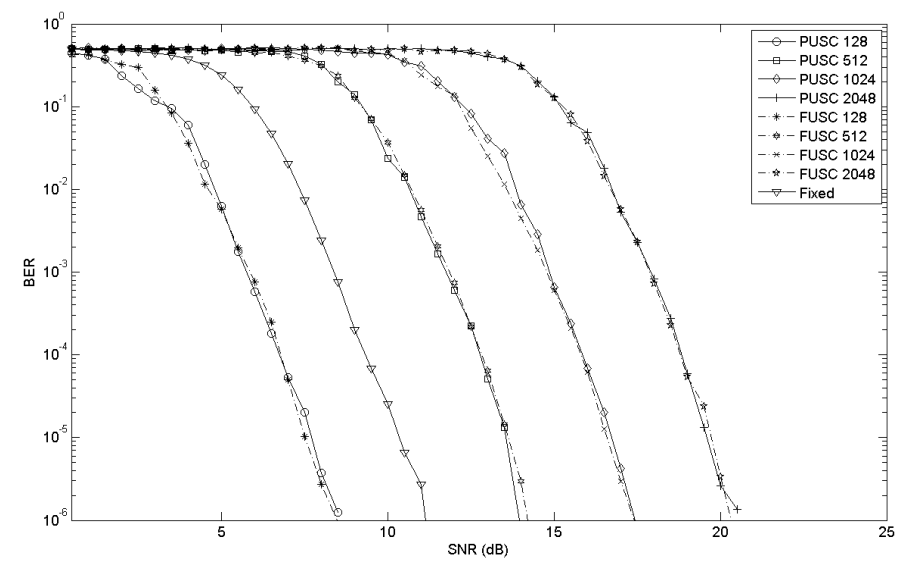

Fig. 2. Mobile versus fixed WiMAX in an AWGN channel 


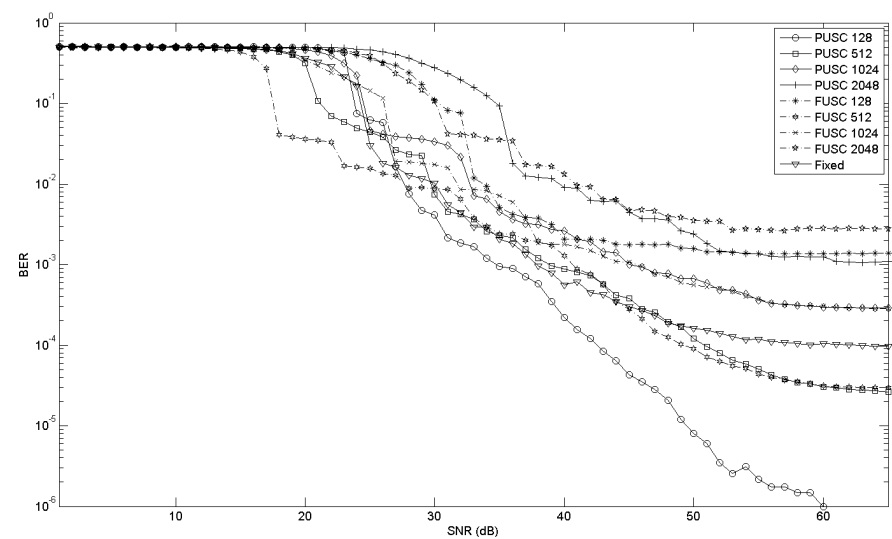

Fig. 3. Mobile versus fixed WiMAX in a Rayleigh channel

\section{B. Effect of physical-layer parameters on performance}

In this section, the influence of physical-layer parameters on mobile WiMAX performance is investigated. In this paper, we limit ourselves to discussing the effect of constellation and FEC coding rate. Fig. 4 shows BER as function of SNR for different constellations in an AWGN channel. The following physical-layer parameters are fixed: the FFT size is 128 , the FEC coding rate is $3 / 4$, and a FUSC permutation is used. The constellation has a large impact on mobile WiMAX performance: the required SNR at a constant BER of $10^{-6}$ increases with about $7 \mathrm{~dB}$ when the constellation is changed from QPSK to 16-QAM and equally when it is changed from 16-QAM to 64-QAM. Fig. 5 shows BER as function of SNR for a 16-QAM constellation with FEC coding rates of $1 / 2$ and $3 / 4$ (the other physical-layer parameters are the same as for Fig. 4). The required SNR increases with $4 \mathrm{~dB}$ at BER $10^{-6}$ if the FEC coding rate is changed from $1 / 2$ to $3 / 4$.

For fixed WiMAX, the required SNR at BER $10^{-6}$ increases with about $6 \mathrm{~dB}$ when going from QPSK to 16-QAM, and equally when going from 16-QAM to 64-QAM. Also, increasing the FEC coding rate from $1 / 2$ to $3 / 4$ means an increase of $3.5 \mathrm{~dB}$ for the required SNR at BER $10^{-6}$. We conclude that the influence of constellation and coding rate on performance is very similar for fixed and mobile WiMAX.

\section{MIMO performance}

In this section, we investigate the BER versus SNR performance of multiple transmitting and receiving antennas on a mobile WiMAX system. Fig. 6 shows BER versus SNR graphs for SISO, a 2x1 Alamouti MISO diversity scheme, and a 2x2 Alamouti MIMO diversity scheme in the SUI 4 channel. For this figure, the following physical-layer parameters are chosen: constellation is QPSK, FEC coding rate is $1 / 2$, CP length is $1 / 32$, FFT size is 128 , and the subcarrier permutation is FUSC. At a BER of $10^{-6}$ and when compared to the SISO configuration, diversity gains (i.e., reductions in required SNR) of $1.5 \mathrm{~dB}$ and $5.5 \mathrm{~dB}$ are obtained for the MISO and MIMO system, respectively.

In literature, the diversity gain for a $2 \times 2$ MIMO system at

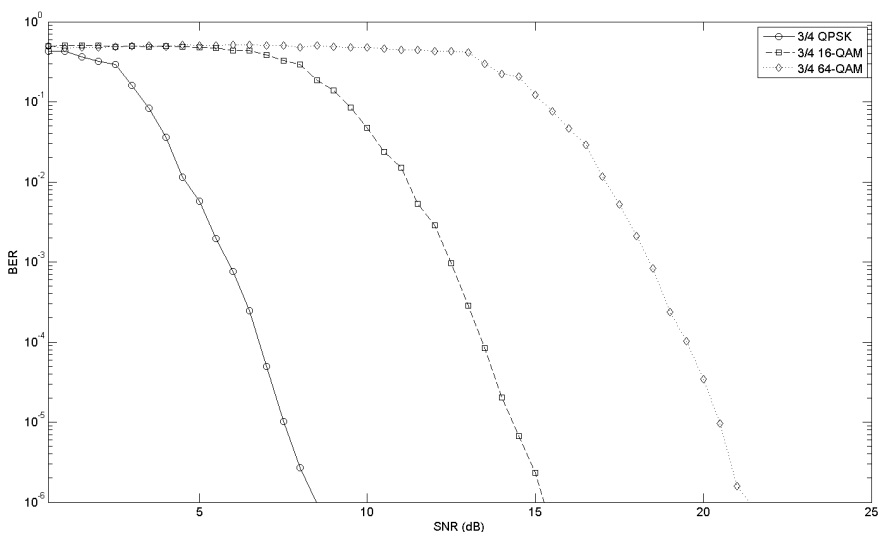

Fig. 4. Influence of constellation in an AWGN channel

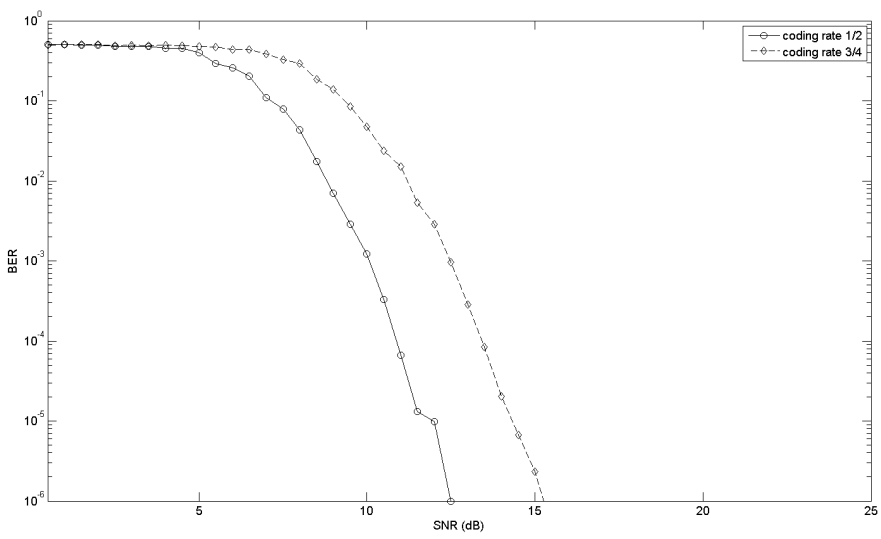

Fig. 5. Influence of coding rate in an AWGN channel

a BER of $10^{-4}$ is investigated in [8] and [9]. These works report diversity gains between 7 and $7.5 \mathrm{~dB}$. For comparison, Fig. 6 shows a slightly lower gain of $5.5 \mathrm{~dB}$ at BER $10^{-4}$ for the $2 \times 2$ MIMO configuration. Also for a $2 \times 2$ MIMO system, the required SNR for BER $10^{-4}$ is found to be $18 \mathrm{~dB}$ in [10], which agrees well with our obtained value of $19 \mathrm{~dB}$. In [11], the required SNR for a BER of $10^{-3}$ for a $2 \times 2$ MIMO system is investigated for different constellations: the required SNRs are $10 \mathrm{~dB}$ for QPSK, $16.6 \mathrm{~dB}$ for 16-QAM, and $22.4 \mathrm{~dB}$ for 64-QAM. From Fig. 6, we obtained required SNRs of 10, 16, and $20 \mathrm{~dB}$, respectively. We conclude that our results compare well to the MIMO results in literature.

\section{Link budget and achievable range}

In this section, a realistic business modem scenario is considered. A link budget is constructed to determine the achievable physical-layer bitrate versus its range. For the link budget, the following properties of the WiMAX system are chosen: the heights of the base station (BS) and mobile station (MS) are $30 \mathrm{~m}$ and $6 \mathrm{~m}$, respectively. The input power to the BS antenna is $35 \mathrm{dBm}(3.2 \mathrm{~W})$, which is a typical value for Belgium. The gains of the BS and MS antennas are $17 \mathrm{dBi}$ and $18 \mathrm{dBi}$, respectively. The MS antenna is a fixed antenna that is placed outdoor. The center frequency is $2500 \mathrm{MHz}$. 


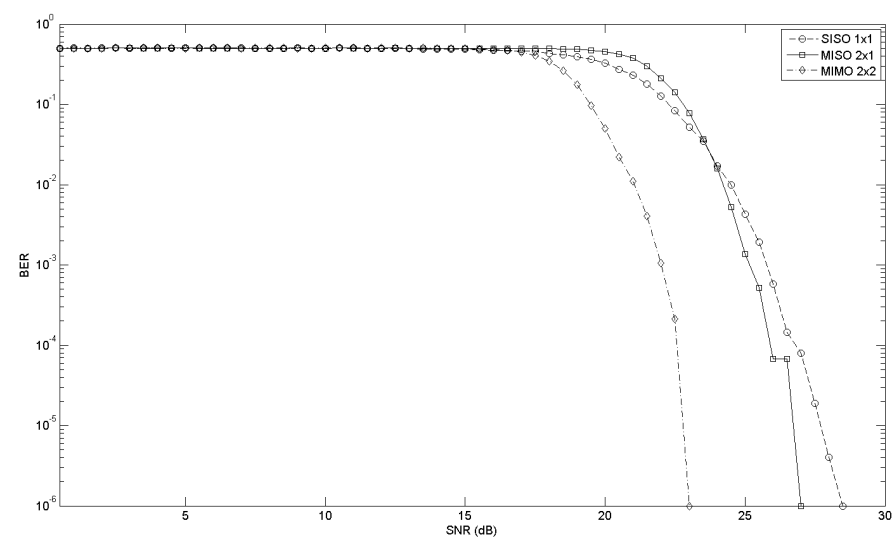

Fig. 6. BER versus SNR graphs for multiple antenna diversity schemes

For calculation of the range, the Erceg-B path loss model is used [6]. A shadowing margin is taken into account for a coverage requirement of $90 \%$ on the edge of the cell. Furthermore, a margin of $10 \mathrm{~dB}$ for temporal fading [3] and an interference margin of $2 \mathrm{~dB}$ are considered [12].

Fig. 7 shows the physical-layer bitrates versus their corresponding range using the SNR requirements at BER $10^{-6}$ in an AWGN channel. For the mobile WiMAX modes, the bitrates are calculated for RF channel bandwidths equal to multiples of $1.25 \mathrm{MHz}$ (associated sampling factor of 28/25). For the fixed WiMAX mode, the bitrates are derived for an RF channel bandwidth of $2.5 \mathrm{MHz}$ (associated sampling factor of 144/125). These settings result in a subcarrier frequency spacing of $11.25 \mathrm{kHz}$ for fixed WiMAX, comparable to the constant value of $10.94 \mathrm{kHz}$ for mobile WiMAX. The maximum mobile WiMAX range is $3.8 \mathrm{~km}$ with a bitrate of 0.76 Mbps (PUSC, FFT size 128). The maximum achievable mobile WiMAX bitrate is $73.3 \mathrm{Mbit} / \mathrm{s}$ and reaches only $0.5 \mathrm{~km}$ far (FUSC, FFT size 2048). For the fixed WiMAX mode, the maximum range is $2.9 \mathrm{~km}$ and the maximum bitrate is 9.2 Mbps. From these results, it is clear that mobile WiMAX can achieve both higher physical bitrates and larger ranges compared to fixed WiMAX.

\section{PERFORMANCE RESUlTS FOR A REAL ENVIRONMENT}

\section{A. Tapped delay line model}

We investigate the performance of a mobile WiMAX system in a specific geographical area: the Zuiderpoort area in the city of Ghent, Belgium. A satellite photo of the area is shown in Fig. 8. We first develop a model for delay dispersion of propagation paths in this specific area, a phenomenon which has a detrimental effect on BER performance. To this end, raytracing simulations of the propagation environment are performed in the Wireless Insite ${ }^{\circledR}$ software package.

Four configurations are considered. Two possible locations for the transmitting antenna (Tx) are investigated: the $\mathrm{Tx}$ is either installed at a height of $15 \mathrm{~m}$ at location 1 in Fig. 9(a)), or at a height of $45 \mathrm{~m}$ at location 2. Both Tx locations are situated on the roof of local office buildings. The receiving antenna

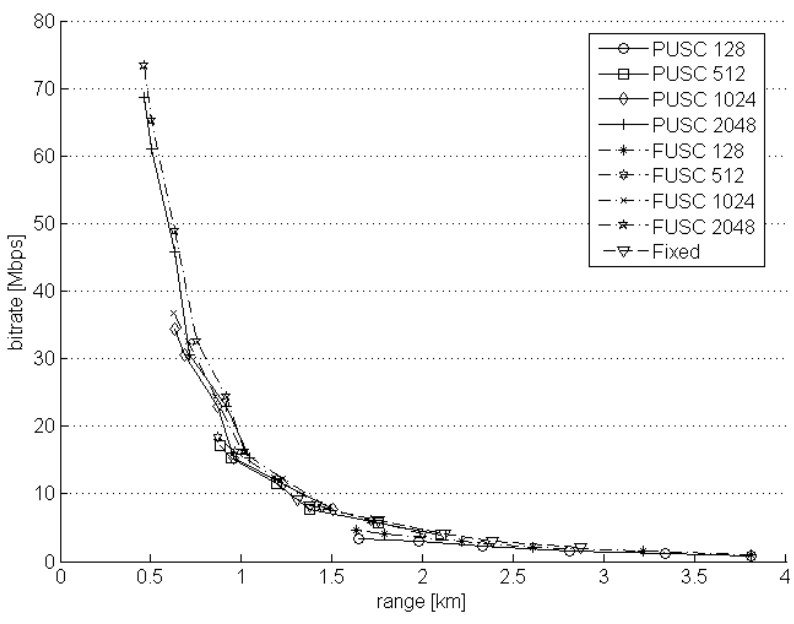

Fig. 7. Bitrate versus range for an AWGN channel

$(\mathrm{Rx})$ is considered to be near ground level: two heights of $1.5 \mathrm{~m}$ and $2.5 \mathrm{~m}$ are investigated. Receiving antennas $(\mathrm{Rx})$ are placed on the different points of a grid laid over the area. The grid is shown in Fig. 9(b)). Two adjacent receiving antennas are spaced $5 \mathrm{~m}$ apart. Narrowband simulations are performed at a center frequency of $2500 \mathrm{MHz}$ and omnidirectional, vertically polarized dipole antennas are used for both the Tx and the Rx.

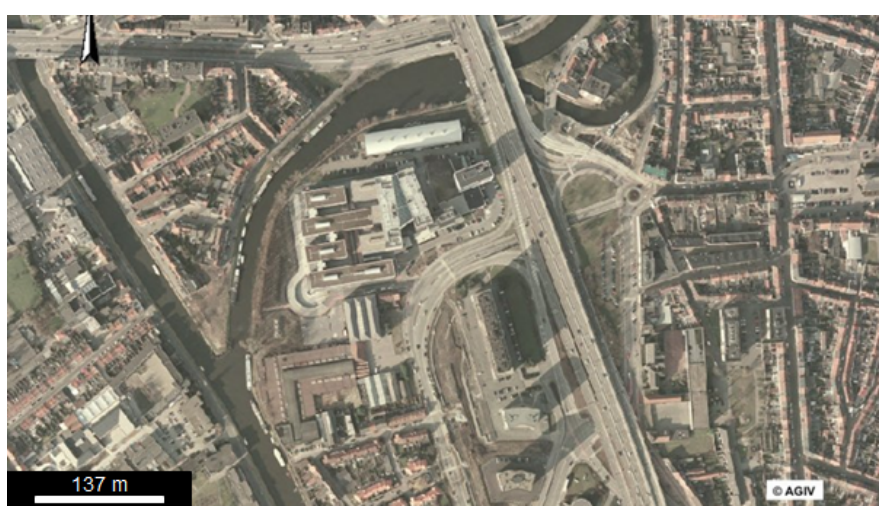

Fig. 8. Zuiderpoort area in Ghent, Belgium

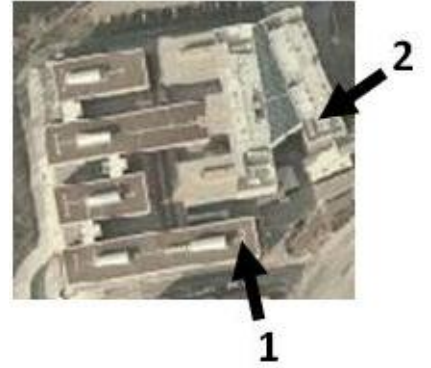

(a)

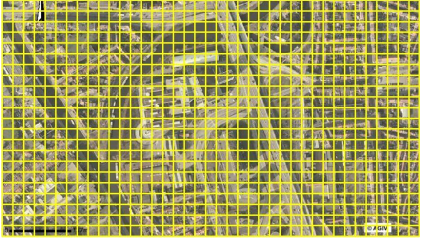

(b)
Fig. 9. Transmitters (a) and receivers (b) in the Zuiderpoort area 
A 3-D model of the building infrastructure in the Zuiderpoort area is imported into Wireless Insite ${ }^{\circledR}$. Next, the propagation paths created by reflections and diffractions off the buildings' surfaces and edges are simulated. The delay and received power of each propagation path is used to construct a tapped delay line channel model which accounts for the delay dispersion in the environment.

The tapped delay model is constructed as follows. First, the maximum delay of propagation paths is determined and found equal to $4.35 \mu \mathrm{s}$. Next, the delay axis from $0 \mu \mathrm{s}$ to $4.35 \mu \mathrm{s}$ is subdivided into three delay bins of equal width. Based on their delay, the simulated propagation paths are grouped into these delay bins. For each Rx position on the grid, the tapped delay line model consists of three taps with delays corresponding to the delay bin centers, and powers corresponding to the sum of received powers of all propagation paths in the considered delay bin. The final tapped delay line model is constructed by linear averaging of tap powers over all Rx positions on the receiver grid. In Table I, the tapped delay line models for the four configurations (two Tx heights and two Rx heights) are given. In an additional step, the tap powers are normalized to the power of the first tap.

In Table I, the lowest delay dispersion is associated with the largest Tx and Rx antenna heights, i.e., $45 \mathrm{~m}$ and $2.5 \mathrm{~m}$, respectively. This means that for this configuration the received signal's power is concentrated at smaller delays because of fewer obstructions in the line-of-sight path between the Tx and Rx. This can also be observed from Fig. 10 and Fig. 11, which show simulated propagation paths in the Zuiderpoort area for a certain $\mathrm{Rx}$ position on the grid and Tx heights of $15 \mathrm{~m}$ and $45 \mathrm{~m}$, respectively. For the lowest Tx height of $15 \mathrm{~m}$ in Fig. 10, most propagation paths undergo several reflections and diffractions before arriving at the Rx. Contrarily, in Fig. 11 for the higher $\mathrm{Tx}$ at $45 \mathrm{~m}$, propagation paths undergo less interactions with the environment because of less obstructions, meaning a lower delay dispersion of paths for the higher Tx.

\begin{tabular}{|l|l|l|l|l|}
\hline Tx 15m, Rx 1.5m & Tap 1 & Tap 2 & Tap 3 & Unit \\
\hline Delay & 0.72 & 2.17 & 3.62 & $\mu \mathrm{s}$ \\
\hline Power & 0 & -13 & -35 & $\mathrm{~dB}$ \\
\hline \hline Tx 45m, Rx 1.5m & Tap 1 & Tap 2 & Tap 3 & Unit \\
\hline Delay & 0.72 & 2.17 & 3.62 & $\mu \mathrm{s}$ \\
\hline Power & 0 & -18 & -38 & $\mathrm{~dB}$ \\
\hline \hline Tx 15m, Rx 2.5m & Tap 1 & Tap 2 & Tap 3 & Unit \\
\hline Delay & 0.72 & 2.17 & 3.62 & $\mu \mathrm{s}$ \\
\hline Power & 0 & -13 & -33 & $\mathrm{~dB}$ \\
\hline \hline Tx 45m, Rx 2.5m & Tap 1 & Tap 2 & Tap 3 & Unit \\
\hline Delay & 0.72 & 2.17 & 3.62 & $\mu \mathrm{s}$ \\
\hline Power & 0 & -19 & -38 & $\mathrm{~dB}$ \\
\hline \multicolumn{5}{|l}{ TABLE I } \\
\hline
\end{tabular}

TAPPED DELAY LINE MODEL FOR FOUR CONFIGURATIONS IN THE ZUIDERPOORT AREA

\section{B. Link budget and achievable range}

Table II shows the results of a link budget calculation for determination a mobile WiMAX system's range in the

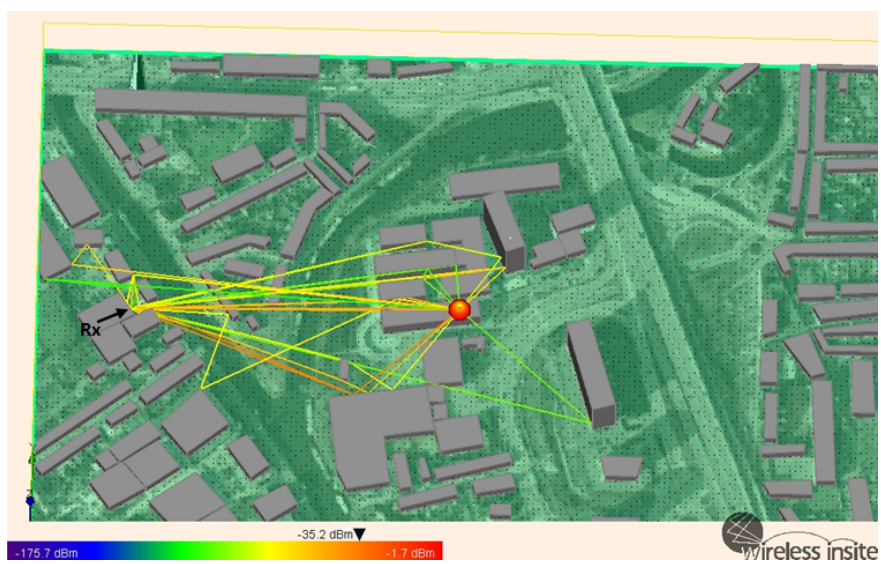

Fig. 10. Propagation paths between the Tx at $15 \mathrm{~m}$ height and an $\mathrm{Rx}$ position

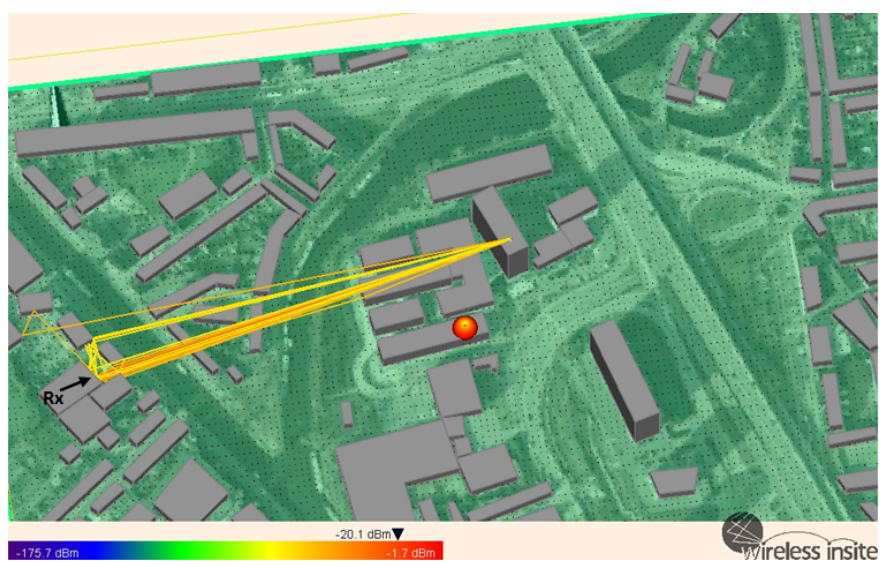

Fig. 11. Propagation paths between the $\mathrm{Tx}$ at $45 \mathrm{~m}$ height and an $\mathrm{Rx}$ position

Zuiderpoort area. The mobile WiMAX system parameters are the same as in Section III-D, expect the Tx and Rx heights are chosen in accordance with the four configurations in Section IV-A. The path loss model used for range calculation is the Erceg-B model [6]. The tapped delay line models in Table I are used in the Simulink ${ }^{\circledR}$ model of Fig. 1 to determine the required SNRs at BER $10^{-6}$. These SNR requirements are then converted to achievable ranges using the link budget. The maximum achievable range (lowest modulation scheme) for each of the four configurations is given in Table II. For comparison, the maximum achievable range for an AWGN channel is also shown. The largest range is obtained with the Tx at a height of $45 \mathrm{~m}$ and the $\mathrm{Rx}$ at a height of $2.5 \mathrm{~m}$. The ranges for the AWGN channel are larger than the ranges for the tapped delay line model. This is expected as the AWGN channel does not account for multipath fading as opposed to the tapped delay line models.

\section{MIMO performance}

In this section, the performance of a $2 \times 2$ MIMO system is compared to that of a SISO system for the tapped delay line models in Table I. The following physical-layer parameters are chosen: an FFT size of 128, PUSC permutation, and a 


\begin{tabular}{|c|c|c|}
\hline Configuration & $\begin{array}{c}\text { Maximum range } \\
\text { AWGN }\end{array}$ & $\begin{array}{c}\text { Maximum range } \\
\text { Tapped delay line }\end{array}$ \\
\hline Tx $15 \mathrm{~m}, \mathrm{Rx} 1.5 \mathrm{~m}$ & $1.4 \mathrm{~km}$ & $1.3 \mathrm{~km}$ \\
\hline Tx $15 \mathrm{~m}, \mathrm{Rx} 2.5 \mathrm{~m}$ & $1.6 \mathrm{~km}$ & $1.5 \mathrm{~km}$ \\
\hline Tx $45 \mathrm{~m}, \mathrm{Rx} 1.5 \mathrm{~m}$ & $2.6 \mathrm{~km}$ & $2.4 \mathrm{~km}$ \\
\hline Tx $45 \mathrm{~m}, \mathrm{Rx} 2.5 \mathrm{~m}$ & $3.0 \mathrm{~km}$ & $2.7 \mathrm{~km}$ \\
\hline
\end{tabular}

TABLE II

MAXIMUM ACHIEVABLE RANGES FOR THE ZUIDERPOORT AREA

$\mathrm{CP}$ length of $1 / 32$. For the analysis, the tapped delay line model for a Tx height of $45 \mathrm{~m}$ and an Rx height of $2.5 \mathrm{~m}$ is chosen. Table III shows the required SNR at BER $10^{-6}$ for the different constellations. The difference in SNR requirement between the SISO and the $2 \times 2$ MIMO system ranges from 3 to $3.5 \mathrm{~dB}$. For the 64-QAM constellation with coding $3 / 4$, this difference is even $5 \mathrm{~dB}$. In Section III-C, it was found that the use of a $2 \times 2$ MIMO system leads to an improvement of $5.5 \mathrm{~dB}$ with respect to a SISO system. The SNR gains for the tapped delay line model are only slightly smaller compared to this value. When we look at the maximum achievable ranges in Table IV, we note an improvement of 0.2 to $0.6 \mathrm{~km}$ for the $2 \times 2$ MIMO system compared the SISO system.

\begin{tabular}{|l|c|c|c|}
\hline Constellation & Coding rate & SISO & MIMO \\
\hline QPSK & $1 / 2$ & $7 \mathrm{~dB}$ & $3.5 \mathrm{~dB}$ \\
\hline QPSK & $3 / 4$ & $9 \mathrm{~dB}$ & $6 \mathrm{~dB}$ \\
\hline 16-QAM & $1 / 2$ & $15 \mathrm{~dB}$ & $12 \mathrm{~dB}$ \\
\hline 16-QAM & $3 / 4$ & $19 \mathrm{~dB}$ & $15.5 \mathrm{~dB}$ \\
\hline 64-QAM & $2 / 3$ & $23 \mathrm{~dB}$ & $19.5 \mathrm{~dB}$ \\
\hline 64-QAM & $3 / 4$ & $26 \mathrm{~dB}$ & $21 \mathrm{~dB}$ \\
\hline
\end{tabular}

TABLE III

REQUIRED SNR AT BER $10^{-6}$ FOR THE TX AT 45 M AND THE RX AT $2.5 \mathrm{M}$ (TAPPED DELAY LINE MODEL)

\begin{tabular}{|l|c|c|c|}
\hline Constellation & Coding rate & SISO & MIMO \\
\hline QPSK & $1 / 2$ & $2.7 \mathrm{~km}$ & $3.3 \mathrm{~km}$ \\
\hline QPSK & $3 / 4$ & $2.4 \mathrm{~km}$ & $2.9 \mathrm{~km}$ \\
\hline 16-QAM & $1 / 2$ & $1.7 \mathrm{~km}$ & $2.1 \mathrm{~km}$ \\
\hline 16-QAM & $3 / 4$ & $1.4 \mathrm{~km}$ & $1.7 \mathrm{~km}$ \\
\hline 64-QAM & $2 / 3$ & $1.1 \mathrm{~km}$ & $1.4 \mathrm{~km}$ \\
\hline 64-QAM & $3 / 4$ & $0.9 \mathrm{~km}$ & $1.2 \mathrm{~km}$ \\
\hline
\end{tabular}

TABLE IV

MAXIMUM ACHIEVABLE RANGES FOR THE TX AT 45 M AND THE RX AT $2.5 \mathrm{M}$ (TAPPED DELAY LINE MODEL)

\section{CONCLUSions}

In this paper, the performance of a mobile WiMAX system is investigated for different settings of its physical-layer parameters and for realistic propagation channels. For this, a model for the physical layer of IEEE $802.16 \mathrm{e}$ is developed in Simulink ${ }^{\circledR}$. Because of the adjustable number of subcarriers and new permutation schemes offered by mobile WiMAX, simulations show that lower signal-to-noise ratio requirements and thus larger achievable cell ranges are possible compared to fixed WiMAX, albeit at the expense of bitrate. Of the mobile WiMAX physical-layer parameters, constellation has the greatest effect on signal-to-noise ratio performance, followed by coding rate. A $2 \times 2$ MIMO system with diversity coding simulated for the SUI 4 propagation channel model is found to lower the signal-to-noise ratio requirement with $5.5 \mathrm{~dB}$.

A tapped delay line channel model is developed for a specific area in Ghent (Belgium) using the Wireless Insite ${ }^{\circledR}$ raytracing software. For this area, the maximum achievable range of a realistic mobile WiMAX system is found to be $2.7 \mathrm{~km}$. For this environment, a $2 \times 2$ MIMO diversity scheme lowers the signal-to-noise ratio requirement with 3 to $3.5 \mathrm{~dB}$.

\section{ACKNOWLEDGMENT}

Wout Joseph is a Post-Doctoral Fellow of the FWO-V (Research Foundation - Flanders). Daan Pareit would like to thank the IWT-Vlaanderen (Institute for the Promotion of Innovation through Science and Technology in Flanders) for financial support through his Ph.D. grant.

\section{REFERENCES}

[1] Air Interface for Fixed Broadband Wireless Access Systems, IEEE 802.16 Working Group on Broadband Wireless Access Standards, October 2004, ref.: IEEE Std 802.16-2004, online: www.ieee802.org/16.

[2] W. Joseph, W. Reynders, J. Debruyne, and L. Martens, "Influence of Channel Models and MIMO on the Performance of a System based on IEEE 802.16," in IEEE Wireless Communications and Networking Conference (WCNC), Kowloon, CN, March 2007, pp. 1826-1830.

[3] W. Joseph and L. Martens, "Performance Evaluation of Broadband Fixed Wireless System based on IEEE 802.16," in IEEE Wireless Communications and Networking Conference (WCNC), Las Vegas, NV, US, April 2006, pp. 978-983.

[4] V. Erceg et al., Channel Models for Fixed Wireless Applications, IEEE 802.16 Broadband Wireless Access Working Group, 2003.

[5] Air Interface for Fixed and Mobile Broadband Wireless Access Systems - Amendment 2: Physical and Medium Access Control Layers for Combined Fixed and Mobile Operation in Licensed Bands, IEEE 802.16 Working Group on Broadband Wireless Access Standards, February 2006, ref.: IEEE Std 802.16e-2005, online: www.ieee802.org/16.

[6] V. Erceg, L. J. Greenstein, S. Y. Tjandra, S. R. Prakoff, A. Gupta, B. Kubic, A. A. Julius, and R. Bianchi, "An Empirically Based Path Loss Model for Wireless Channels in Suburban Environments," IEEE Journal on Selected Areas in Communications, vol. 17, no. 2, pp. 1205-1211, July 1999.

[7] S. M. Alamouti, "A Simple Transmit Diversity Technique for Wireless Communications," IEEE Journal on Selected Areas in Communications, vol. 16, no. 8, pp. 1451-1458, October 1998.

[8] E. Biglieri, A. Goldsmith, B. Muquet, and H. Sari, "Diversity, Interference Cancellation and Spatial Multiplexing in MIMO Mobile WiMAX Systems," in IEEE Mobile WiMAX Symposium, Orlando, FL, US, March 2007, pp. 74-79.

[9] A. Doufexi, S. Armour, A. Nix, and M. Beach, "Evaluating the Physical Layer Performance of an OFDM candidate for Fourth Generation Networks," in IST Mobile Communications Summit, Thessaloniki, GR, June 2002.

[10] D. Marabissi, F. Meucci, L. Pierucci, and L. S. Ronga, "Adaptive Selection of MIMO Schemes in IEEE 802.16e," in International Conference On Communications And Mobile Computing, Honolulu, HI, US, August 2007, pp. 423-428.

[11] S. Sezginer and H. Sari, "A High-Rate Full-Diversity $2 \times 2$ Space-Time Code with Simple Maximum Likelihood Decoding," in IEEE International Symposium on Signal Processing and Information Technology, Giza, EG, December 2007, pp. 1132-1136.

[12] WiMAX Forum, Mobile WiMAX - Part I: A Technical Overview and Performance Evaluation, August 2006. 